\title{
The Effectiveness of Voice Therapy for Teachers with Dysphonia
}

\author{
E. Niebudek-Bogusz ${ }^{a} \quad$ B. Sznurowska-Przygockab ${ }^{\text {b. }}$ Miszer ${ }^{a} \quad$ P. Kotyło $^{a}$ \\ A. Sinkiewicz ${ }^{c}$ M. Modrzewskad M. Sliwinska-Kowalska ${ }^{a}$ \\ ${ }^{a}$ Department of Audiology and Phoniatrics, Nofer Institute of Occupational Medicine, Lodz, \\ ${ }^{\mathrm{b}}$ Regional Centre of Occupational Medicine, Gdansk, 'Regional Audiology and Phoniatrics Outpatient Clinic, \\ Bydgoszcz, and ${ }^{\mathrm{d}}$ Phoniatrics Outpatient Clinic, Staszow, Poland
}

\section{Key Words}

Voice therapy $\cdot$ Dysphonia $\cdot$ Teachers

\begin{abstract}
Objective: An incorrect voice emission is a risk factor for developing occupational voice disorders. The study aimed at assessing the effectiveness of voice therapy in female teachers with dysphonia. Methods: The study comprised 133 subjects with voice disorders, taking part in a vocal training programme. A reference group for the present study included 53 teachers with dysphonia. Questionnaire surveys, phoniatric examination and videostroboscopic evaluation were conducted at initial and control examination. Results: In the study group, an improvement after the vocal training was noted in most of the reported symptoms and also in some quantitative parameters of phoniatric examinations compared to the findings for the reference group. The number of patients who assessed their voice as normal increased significantly after the vocal training (2.3 vs. $46.6 \%)$. A significant increase in the mean maximum phonation time, from 13.3 to $16.6 \mathrm{~s}$, was observed. The same applied to voice frequency range (increase from 171 to $226.8 \mathrm{~Hz}$ ). Conclusions: The outcomes of vocal training, such as a subjective improvement of voice quality and an increase in the quantitative parameters (prolonged maximum phonation time, extended voice range) seem to be important parameters for monitoring the effectiveness of training in correct voice emission.
\end{abstract}

Copyright $\odot 2008$ S. Karger AG, Basel
(C) 2008 S. Karger AG, Basel

$1021-7762 / 08 / 0603-0134 \$ 24.50 / 0$

Fax +41613061234

E-Mail karger@karger.ch

www.karger.com

\section{Introduction}

The prevalence of voice disorders is higher among teachers who intensively use their voice at work than in other professional groups whose activities do not involve increased voice effort $[1,2]$. Large-scale US studies revealed that teachers were significantly more likely than non-teachers to present multiple voice symptoms including hoarseness, increased vocal fatigue over a long period of voicing and loss of their singing range [3].

The findings of the study by Smith et al. [4] revealed that over $38 \%$ of the teachers complained of occupationrelated voice disorders, and 39\% of those had to quit teaching activities as a result of voice problems. One should note, however, that only a few people in this professional group presented any basic knowledge of vocal hygiene or the principles of correct voice emission. Clinical examinations with laryngovideostroboscopy indicated hyperfunctional dysphonia, vocal nodules, polypoid hypertrophy and vocal fold oedema as the most prevalent voice pathologies in this population [5].

In Poland, voice disorders have been the most common occupational disease and concerned mainly female teachers, mainly because as much as $79 \%$ of the teachers' population in Poland are women [6]. In Polish studies performed on randomly selected groups of female teachers and female office workers (control group), voice problems were reported by $69 \%$ female teachers and $36 \%$ nonteachers. Clinical studies, including videostroboscopic

Ewa Niebudek-Bogusz, MD, $\mathrm{PhD}$

Department of Audiology and Phoniatrics, Nofer Institute of Occupational Medicine ul. Teresy 8

PL-91-348 Lodz (Poland)

Tel. +48 42631 4545, Fax +48 42631 4519, E-Mail ebogusz@imp.lodz.pl 
examination, revealed work-related dysphonia in about $32.7 \%$ of the teachers versus $9.6 \%$ of the control group [7]. Voice problems applied not only to professionals with a long employment time but also to young teachers and students at teachers' college [8].

One of the main factors accounting for the high prevalence of voice disorders among teachers seems to be the lack of training in voice emission techniques during their university studies. Some evidence for this conclusion derives from the results of applying vocal training in the clinical treatment of patients with professional dysphonia [9-12]. It is important that the training in correct voice emission for future teachers be introduced as early as at the stage of college education [13]. Our earlier study on this particular problem confirmed that vocal training administered to the students of pedagogy helped them significantly improve their voice quality [14].

Although the benefits resulting from the prevention of voice disorders and the correction of voice emission have been widely recognized, no Polish study to estimate the effectiveness of such a therapy in work-related voice disorders could be traced in relevant literature. The aim of the present project was to evaluate the effectiveness of voice therapy in teachers who were referred to a phoniatrician because of a chronic dysphonia.

\section{Subjects and Methods}

\section{Subjects}

The study was performed in collaboration with 4 phoniatric outpatient clinics in Poland. Altogether, the study covered $186 \mathrm{fe}-$ male teachers aged $23-60$ years (mean age $=38.7$ ) who complained of voice disorders and were referred to a phoniatric centre. The study concerned only female subjects because the teaching profession is highly feminized in Poland, as mentioned above. The studied subjects experienced vocal effort for $18-30 \mathrm{~h}$ a week (mean = 25). Most of them (approx. 80\%) were primary and secondary school teachers, the others worked at colleges and universities. All the subjects were briefed on the purpose of the vocal training programme and encouraged to participate. A complete voice training programme was attended by 133 of the subjects (group I - 'voicetrained teachers'). For various non-medical reasons, 53 of the subjects could not undergo this therapy and became the controls (group II - 'non-voice-trained teachers') for the study. The mean age and employment time were similar in both groups (table 1). A questionnaire survey, and laryngological, phoniatric and videostroboscopic examinations were performed before and after voice therapy in the study group, as well as at an initial examination and 3 months afterwards in the control group.

The teachers from both groups received advice on vocal hygiene. They were also prescribed, if necessary, inhalations, humidification and/or vitamins as well as pharmacological treatment for other systemic diseases.

The Effectiveness of Voice Therapy for Teachers
Table 1. Characteristics of the study group (voice-trained teachers) and the control group (non-voice-trained teachers)

\begin{tabular}{lcll}
\hline & $\begin{array}{l}\text { Sub- } \\
\text { jects }\end{array}$ & $\begin{array}{l}\text { Mean age } \pm \text { SD } \\
\text { years }\end{array}$ & $\begin{array}{l}\text { Mean employment } \\
\text { time } \pm \text { SD, years }\end{array}$ \\
\hline $\begin{array}{l}\text { Group I (voice- } \\
\text { trained teachers) }\end{array}$ & 133 & $\begin{array}{l}38.7 \pm 6.9 \\
(\text { range }=23-60)\end{array}$ & $\begin{array}{l}14.5 \pm 6.8 \\
(\text { range }=1-35)\end{array}$ \\
\hline $\begin{array}{l}\text { Group II (non-voice- } \\
\text { trained teachers) }\end{array}$ & 53 & $\begin{array}{l}38.2 \pm 6.6 \\
(\text { range }=25-57)\end{array}$ & $\begin{array}{l}14.5 \pm 6.6 \\
(\text { range }=2-32)\end{array}$ \\
\hline
\end{tabular}

\section{Methods}

Questionnaire

The questionnaire included items regarding the patient's age, intensity and duration of work-related vocal effort [type of school and the subject taught, teaching hours per week (at least $18 \mathrm{~h} /$ week - inclusion criterion); years of working as a teacher], and subjective vocal symptoms appearing over the working life: e.g. recurring or chronic hoarseness, its association with vocal effort, frequent voicelessness, vocal fatigue (voice tiredness), sensation of dry throat or lump in the pharynx and dry cough. The patients were inquired about other conditions that could affect correct phonation (such as e.g. nose and sinus diseases), tobacco smoking habits and current pharmacological treatment. The questionnaire consisted of 30 items including 10 yes/no questions on vocal symptoms. The subjective effectiveness of voice therapy was assessed based on the patient's perception of his voice condition expressed as 'no improvement', 'noticeable improvement but the voice is still incorrect' (dysphonic condition) or 'considerable improvement; the voice is thought to be correct' (euphonic). Furthermore, in the control examination, the patients compared each of the reported initial vocal symptoms on a binary scale: $0=$ no or poor improvement, and $1=$ lack of symptom or considerable improvement. The questionnaire data were collected by a trained nurse, and in individual cases the survey could be extended with additional questions on individual health problems, if this was the case.

\section{Clinical Examination}

All the study participants were subjected to routine otolaryngological examination, including laryngeal pre-assessment with indirect laryngoscopy. Phoniatric examination comprised an assessment of voice quality, breathing technique, correctness of phonation and articulation, resonator activation, presence of nasalization, as well as excessive neck muscle tension that is characteristic of muscle tension dysphonia. The maximum phonation time (MPT) was measured: the subject was asked to phonate a sustained /a:/ vowel at a comfortable pitch and loudness as long as possible during a single exhalation. MPT is a simple aerodynamic parameter and its decrease below 20 s (i.e. the minimum normal MPT value) may contribute to voice disorders. The assessment also included the determination of the mean position of fundamental frequency and its range during speaking (hertz), and the mean voice intensity (decibel).

The videostroboscopic procedure was described exactly elsewhere [7]. For each stroboscopic parameter, a binary scale was used $(0=$ normal, $1=$ abnormal $)$. The records were evaluated by 
2 otolaryngologists/phoniatricians. They were presented without sound; therefore, the evaluation was based only on the visual impression. The focus was on the differences between the initial and control videostroboscopic images recorded for individual subjects. The recording was used not only to evaluate the vocal fold status before and after therapy but also to adapt the vocal training protocol to individual needs.

Vocal Hygiene Programme

On the first visit the subjects received brief information concerning vocal hygiene, which included advice on how to (a) eliminate voice abuse, (b) maintain proper hydration of the upper respiratory tract and (c) avoid irritants such as alcohol or coffee. Also, each patient was provided with a guidebook for teachers with information about: (1) production of normal voice, (2) voice conservation and target behaviours eliminating vocal abuse/misuse, (3) environmental awareness (e.g. voicing in the presence of background noise), (4) maintenance of a healthy lifestyle and (5) illustrated description of vocal training exercises.

\section{Vocal Training Programme}

The main aim of the vocal training programme (for the training protocol see appendix) was to restore normal (euphonic) voice with optimum acoustic characteristics. The persons to conduct the training were 5 logopaedists (speech therapists) from 4 outpatient phoniatric clinics participating in the study. To standardize the vocal training programme each of the trainers had attended a single introductory session before the training started. The vocal training programme comprised 4 main elements: (1) breathing and relaxation exercises, (2) vocal function exercises, (3) resonant improvement exercises and (4) carryover exercises. According to the training protocol, the duration of the vocal training varied between 2 and 4 months; the number of sessions ranging from 9 to 18 , with a session of 45-60 min once or twice a week. At each session, the patient practised the techniques of correct voice emission and then worked on his own at home for 8-10 min daily. All the patients received materials with description of the exercises. The effects of the patient's home performance were evaluated at the beginning of the subsequent session. The patient could move on to the next stage of the training if progress had been positively assessed by the speech therapist.

The training was adapted to individual needs. The effects of voice therapy were evaluated (see appendix) after a minimum of 2 months since the training had started, after at least 8 logopaedic sessions, and after a significant improvement had been noted by the patient and by the logopaedists.

\section{Statistical Analysis}

A stepwise statistical approach was used. At first, the study and control groups were compared with Fisher's exact test for the frequency of the signs and symptoms of voice disorders, as well as the type of voice pathology diagnosed at the initial examination.

The next step was to assess the effectiveness of the vocal training programme by comparing the frequency of favourable changes in subjective symptoms and in objective voice parameters at the initial and final examinations. This was done with the Fisher exact test. Multiple analyses of variance were used for repeated results for the same subjects to assess the changes in the quantitative parameters (phonation time, voice range profile). In all the statistical methods applied, the level of significance was $\mathrm{p}<0.05$.

\section{Results}

\section{Initial Examination}

The questionnaire data revealed that the most prevalent complaints reported by the examined teachers included: throat dryness, voice tiredness, temporary voicelessness and lump-in-throat sensation. The percentage of patients suffering from these problems in the voicetrained and non-voice-trained groups is presented in figure 1 (see initial examination). Furthermore, the initial examination revealed incorrect breathing in $76.3 \%$ of the subjects in group I versus $83.6 \%$ in group II, excessive neck muscle tension in 67.2 versus $77.4 \%$, 'hard' phonation with rough voice in 57.4 versus $60.6 \%$ and incorrect resonator activation in 32.1 versus $28.6 \%$ of the subjects in group II. The following pathological parameters were recorded most frequently at the initial videostroboscopic examination: reduced amplitude of vocal fold vibration (77.9\% of group I and $83.7 \%$ of group II) and incorrect shape of glottal closure (74 vs. $78.6 \%$ ).

Hyperfunctional dysphonia was the most frequently diagnosed pathology. The diagnosis was based on excessive muscle tension of the neck, face and pharynx observed during laryngological examination and accompanying abnormal parameters recorded by videostroboscopy (e.g. diminished amplitude of vocal fold vibration, reduced mucosal wave or glottal hyperclosure with vestibular phonation). In the voice-trained group, hyperfunctional dysphonia was recognised in $67.2 \%$ of the subjects as opposed to $77.4 \%$ in the non-voice-trained group. Furthermore, vocal nodules were detected in $21.4 \%$ of group I and in $37.7 \%$ of group II, and polypoid hypertrophy in 3.1 versus $5.7 \%$ (table 2 ).

At the initial examination, no statistical differences in the incidence of prevailing subjective symptoms were found between the teachers stratified to the study group (voice-trained teachers) and the control group (non-voicetrained teachers). This was also true for the phoniatric and videostroboscopic parameters, except vocal nodules; the latter were more frequent in group II (table 2).

\section{Efficacy of the Vocal Training Programme}

A statistically significant improvement was noted among the voice-trained teachers with respect to most of the examined voice parameters, and the signs and symptoms of voice disorders, while no significant changes were noted among the controls. In the group of teachers receiving voice therapy, a significant decrease was noted in the percentage of patients with chronic hoarseness (by $24.5 \%, \mathrm{p}=0.039$ ), temporary voicelessness (by $47.3 \%, \mathrm{p}<$ 
Fig. 1. Effectiveness of voice therapy in relation to subjective symptoms: voicetrained group versus non-voice-trained group - only the symptoms which differed significantly in the initial and control examinations are presented. $1=$ Chronic hoarseness $(\mathrm{p}=0.039) ; 2=$ temporary voicelessness $(p<0.001) ; 3=$ throat dryness $(\mathrm{p}=0.041) ; 4=$ voice tiredness $(\mathrm{p}<$ $0.001) ; 5=$ aphonia $(\mathrm{p}<0.001) ; 6=$ lumpin-throat sensation $(\mathrm{p}<0.049) ; 7=$ dry cough $(\mathrm{p}=0.046) .{ }^{*} \mathrm{p}<0.05$ : the statistically significant decrease in the percentage of patients with respective symptoms in the control examination.

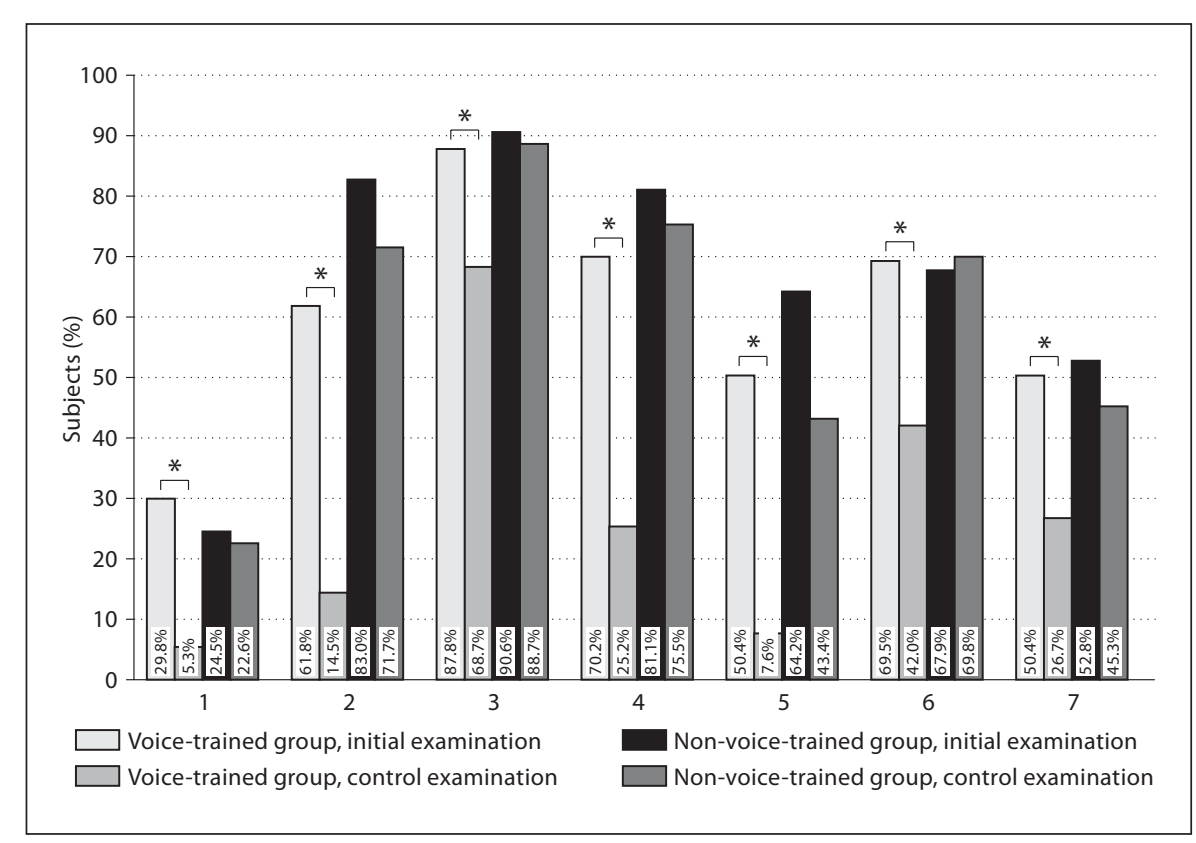

0.001 ) and voice tiredness (by $45 \%, \mathrm{p}<0.001$ ). The frequency of recurring aphonia dropped by approximately $42 \%$ ( $<<0.001$; fig. 1). No improvement of this kind was observed in the control group. After the vocal training, the percentage of subjects who assessed their voice as normal in group I significantly increased $(\mathrm{p}=0.004)$, from $2.3 \%$ (before) to as much as $46.6 \%$ (after). However, in the control group, the comparison of self-estimated improvement in voice quality performed 3 months after the initial examination did not reveal such differences.

The comparison of the results of the control examination in the voice-trained and non-voice-trained teachers revealed that the highest proportion of training-related improvement was associated with a better breathing technique (a $66 \%$ change; $\mathrm{p}<0.001$ ), decreased neck muscle tension ( $\mathrm{a} 46.6 \%$ change, $\mathrm{p}=0.001$ ) and achieving soft phonation (a 54\% change, $\mathrm{p}<0.001$ ).

Differences, indicating improved voice quality in the voice-trained teachers and no improvement in the nonvoice-trained group, were detected for the following quantitative parameters: MPT, mean fundamental frequency and voice intensity (table 3 ), as well as voice frequency range during speaking (fig. 2). At the initial examination, the mean MPT assumed rather low values (13.3 s in the study group, $12.5 \mathrm{~s}$ in the control group). However, in the voice-trained teachers, MPT after vocal training increased by approximately $3 \mathrm{~s}$ (to $16.6 \mathrm{~s}$ ); this difference was statistically significant ( $\mathrm{p}=0.017$; fig. 3 ). Such an improvement was not observed in the control

The Effectiveness of Voice Therapy for Teachers
Table 2. Distribution of subjects, by diagnostic categories

\begin{tabular}{lccc}
\hline Diagnosis & \multicolumn{2}{l}{ Subjects, \% } & \multirow{2}{*}{$\mathrm{p}$} \\
\cline { 2 - 3 } & $\begin{array}{l}\text { group I } \\
(\mathrm{n}=133)\end{array}$ & $\begin{array}{l}\text { group II } \\
(\mathrm{n}=53)\end{array}$ & \\
\hline Hyperfunctional dysphonia & 67.2 & 77.4 & 0.268 \\
Chronic laryngitis & 52.7 & 62.3 & 0.234 \\
Vocal nodules & 21.4 & 37.7 & $0.048^{*}$ \\
Minor polypoid hypertrophy & 3.1 & 5.7 & 0.419 \\
Slight vocal fold abnormalities & 0.8 & 3.8 & 0.171 \\
\hline
\end{tabular}

${ }^{*} \mathrm{p}<0.05$ : the difference between the frequencies in the groups is statistically significant.

group (table 2). A tendency towards a positive change in the fundamental voice frequency and voice intensity could also be observed among the voice-trained teachers (table 3), but these differences were not statistically significant. However, after voice therapy, their voice frequency range (fig. 2) increased significantly, from 171 to $226.8 \mathrm{~Hz}(\mathrm{p}=0.045)$.

Furthermore, in the voice-trained group, the highest proportion of improved videostroboscopic parameters was noted for such parameters as the regularity and amplitude of vocal fold vibration (improvement by 30.5 and $29.8 \%$, respectively), normal mucosal wave (improvement by $28.2 \%$ ) and vocal fold closure (improvement by $30.6 \%$ ). For all the parameters assessed by videostroboscopy, the

Folia Phoniatr Logop 2008;60:134-141 


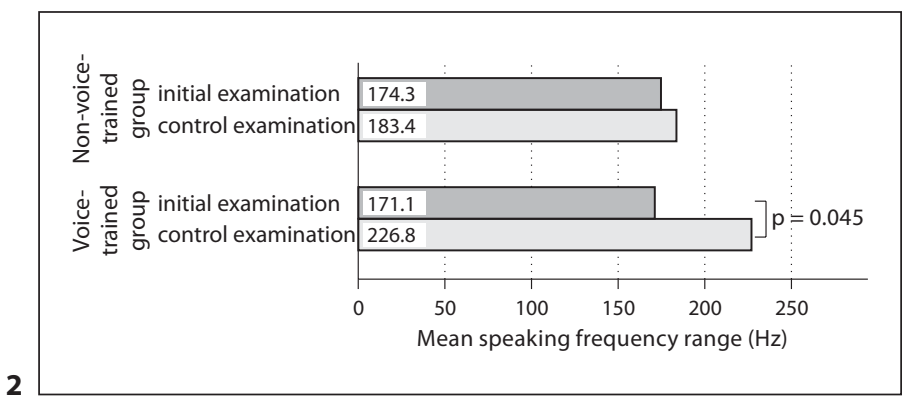

Fig. 2. Voice frequency range: voice-trained group versus nonvoice-trained group.

Fig. 3. Comparison of MPT at the initial and control examinations: voice-trained group versus non-voice-trained group (controls).

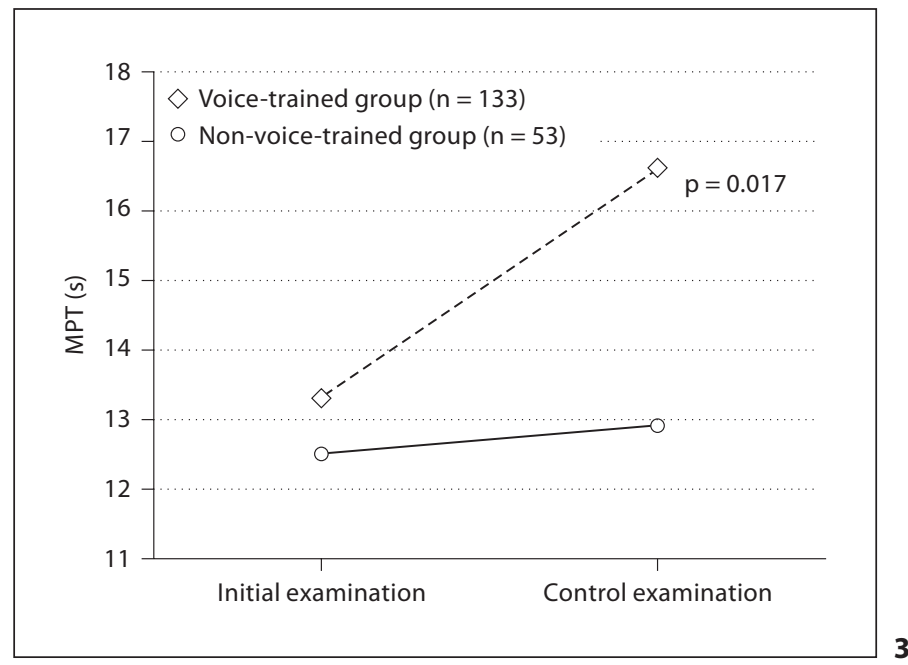

Table 3. Mean phonation time, mean voice frequency and mean voice intensity at the initial and control examinations: voice-trained group (I) versus non-voice-trained group (II)

\begin{tabular}{|c|c|c|c|c|}
\hline & \multicolumn{2}{|c|}{ Group I (n = 133) } & \multicolumn{2}{|c|}{ Group II $(\mathrm{n}=53)$} \\
\hline & $\begin{array}{l}\text { initial } \\
\text { examination }\end{array}$ & $\begin{array}{l}\text { control } \\
\text { examination }\end{array}$ & $\begin{array}{l}\text { initial } \\
\text { examination }\end{array}$ & $\begin{array}{l}\text { control } \\
\text { examination }\end{array}$ \\
\hline Mean MPT, s & 13.3 & $16.6^{*}$ & 12.5 & 12.9 \\
\hline Mean fundamental frequency, $\mathrm{Hz}$ & 199.3 & 203.4 & 200.5 & 200.9 \\
\hline Mean voice intensity, SPL & 63.3 & 64.4 & 60.9 & 61.2 \\
\hline
\end{tabular}

${ }^{*} \mathrm{p}=0.017$ : the difference between MPT before and after voice therapy in the study group is statistically significant.

improvement was statistically significant $(p<0.05)$. For comparison, in the group of non-trained teachers, examined 3 months after the initial examination, an improvement of some of the videostroboscopic parameters was observed as well, but it did not reach statistical significance.

After vocal training, the clinical evaluation of the vocal apparatus revealed an improvement. In the voicetrained group, a decrease was noted in the proportion of subjects with hyperfunctional dysphonia (by ca. $47 \%$, $\mathrm{p}=0.001$ ), in which the recession of the symptoms was evaluated based on the strength of videostroboscopic parameters and a decreased tension of the neck, face and pharynx muscles. Also, the number of subjects with vocal nodules decreased in this group, by $11 \%(\mathrm{p}=0.008)$. In the control group, a comparison of phoniatric findings at the initial and control examinations revealed that although the percentage of subjects with hyperfunctional dysphonia slightly decreased, by ca. $3.8 \%$, the difference was of no statistical significance. In this group, the number of vocal nodules even increased by $5.7 \%$ at the control examination but this difference was not significant either.

\section{Discussion}

The aim of the study was to estimate the effectiveness of voice therapy for teachers with voice disorders. At the initial examination most of the examined subjects showed signs of hyperfunctional dysphonia (above $70 \%$ in the total group). It has been shown that this kind of functional dysphonia (referred to as 'muscle tension dysphonia') is most common among the patients whose jobs involve considerable vocal effort $[15,16]$. Vocal nodules, frequently caused by phonotrauma in individuals professionally using their voice, were detected in $26 \%$ of the total examined teachers. 
Many authors stressed that vocal load in the teaching professions was found to affect the vocal apparatus [1719]. Vilkman [20] suggested that the prevention and treatment of occupational voice disorders calls for improved occupational safety and health arrangements for vocally demanding professions. The present study was undertaken to address this problem. An assumption was made that one of the main factors accounting for voice disorders in teachers is the lack of knowledge about the techniques of correct voice emission. Literature data indicate that vocal training can contribute to improved voice quality and voice endurance in people whose jobs involve considerable vocal load [21-23].

The present study revealed that the highest ratio of subjective improvement after the vocal training referred to the following symptoms: temporary voicelessness, which receded in $47.3 \%$ of the subjects $(\mathrm{p}<0.001)$, and vocal fatigue sensation (voice tiredness), which receded in $45 \%$ of the subjects $(\mathrm{p}<0.001)$. Before the training, only $2.3 \%$ of the female teachers with voice disorders assessed their voice as normal, whereas after the training, this proportion increased to approximately $46.6 \%$ ( $\mathrm{p}=$ $0.004)$. Vocal therapy seems to have been particularly successful in the patients with hyperfunctional dysphonia. The signs of this type of dysphonia were diminished significantly in the voice-trained group $(\mathrm{p}=0.001)$. Other authors also reported a high ratio of effective vocal training in muscle tension dysphonia [24, 25]. Voice therapy seems to be useful also at an early stage of organic dysphonia. After 3-4 months of vocal training, a significant decrease was noted in the prevalence of minor structural vocal fold pathologies, e.g. the percentage of patients with vocal nodules decreased by $11 \%$ in the voice-trained group. Some authors also reported effective voice therapy in patients suffering from minimal benign lesions of the larynx, which prevented phonosurgery $[26,27]$.

The outcomes of vocal training showed an improvement in the quantitative parameters: prolonged MPT and extended voice range. In the study group, decreased MPT before therapy was found in $85 \%$ of the subjects; the shortest MPT value being $6 \mathrm{~s}$. The application of vocal training, including breathing exercises, resulted in the percentage of incorrect results (below $20 \mathrm{~s}$ ) falling down to $73.3 \%$, and MPT prolonged by $3 \mathrm{~s}$ on average ( $\mathrm{p}=$ 0.017). Such changes were not observed in the control group. Similar findings [14], confirming the efficacy of voice therapy, were also obtained in earlier studies performed on students of pedagogy. In this population, the administration of vocal training contributed to a prolonged MPT (by about 6 s on average) and a change in the voice range, by approximately 1.5 tones on average. In the present study, the mean voice range extended significantly, by $56 \mathrm{~Hz}$ in the voice-trained group $(\mathrm{p}=0.045)$, which would indicate a significant improvement in overall voice capabilities.

Although the efficacy of voice therapy may depend on the clinician's attitude to the patient, his personality and the therapist-patient relationship [28,29], the vocal training performed in a similar way by 5 logopaedists from 4 phoniatric clinics appeared to be effective in the examined group. These results are consistent with the findings by Speyer et al. [30]. In order to avoid a therapist-related effect, they evaluated the effectiveness of treatment conducted by 68 speech therapists (no more than 2 patients could be referred to the same therapist). They found a significant improvement in the voice range profile (phonetogram) when comparing the pre- and post-treatment data from all the centres.

Some studies [11,31,32], including prospective randomised clinical trials to compare 2 approaches: direct - vocal training, and indirect - vocal hygiene education, indicated a significant improvement in voice quality mainly with respect to the direct treatment. Our results confirm these findings and make it possible to conclude that vocal training can be useful for the treatment of work-related voice disorders. This is essential when we consider the social and economic costs related to the sickness absence from this group of diseases. Thibeault et al. [33] reported that in the USA alone, the social cost incurred by voice-related absenteeism and treatment expenses in teachers have been estimated to be USD 2.5 billion annually. To solve this problem, effective prevention and rehabilitation programmes need to be developed. Extensive educational activities concerning vocal hygiene should be coupled with correct emission techniques. The costs connected with these methods are much lower than the expenses which would be required otherwise for the treatment of teachers with dysphonia.

\section{Conclusions}

Our results indicate that vocal training is an effective treatment for teachers with voice disorders. A subjective improvement of voice quality and an increase in the quantitative parameters (prolonged MPT, extended voice range) seem to be important parameters for monitoring the effectiveness of vocal training. Moreover, voice therapy has been promoted as the major method used to enhance the efficiency of professional voice use. 


\section{Appendix}

\section{Treatment protocol}

I Discussing the patient's vocal function assessment based on clinical and videostroboscopic findings (the patient, the phoniatrician and the speech therapist).

II Vocal hygiene approach

- Providing information to the patient, including advice on how to (1) eliminate voice abuse directly related to voice use, e.g. yelling, and non-related, e.g. smoky environment, (2) ensure proper body hydration (e.g. drinking 8-10 glasses of water and having it handy while teaching), (3) maintain proper environmental humidity, and (4) avoid irritants (e.g. alcohol, coffee).

- Guidebook for teachers entitled: Voice - The Teacher's Tool of Work with information on (1) production of normal voice, (2) voice conservation and target behaviours eliminating vocal abuse/ misuse, (3) environmental awareness (e.g. voicing in the presence of background noise), (4) maintenance of healthy lifestyle, (5) vocal nodules and other voice disorders in the context of suspected occupational aetiology, and (6) illustrated description of vocal training exercises.

\section{Vocal training protocol}

III Setting the vocal training scheme

The training described below is conducted by a speech therapist and varies depending on the patient's needs. The period of vocal training is between 2 and 4 months; the number of sessions ranging from 9 to 18 , with a single session of $45-60$ min per week. At each session, the patient practises techniques of correct voice emission and then works on his own at home for 8-10 min daily. The effects of the patient's home performance are evaluated at the beginning of the next session. The patient can move on to the next stage of the training once his progress has been positively assessed by the speech therapist.

IV Breathing exercises (3-6 sessions)

a Lying-down position exercises that begin with relaxed breathing and involve breathing support by the diaphragm. The patient controls the function of the intercostal and diaphragm muscles during breathing by watching the movement of a book placed on his abdomen.

b Standing-up position exercises that begin with general muscle relaxation (e.g. by shaking and loosening the limbs and the torso) and involve relaxed breathing with diaphragm support. The patient controls the function of the intercostal and diaphragm muscles during relaxed breathing by watching the movement of his hands placed bilaterally in the waist line.

The following exercises are also performed in the standing-up position

c Relaxed abdominal breathing exercises combined with the voicing of sustained consonants 's', 'f and 'ch' followed by voicing of a vowel combination 'aoeaoeaoe'. During these exercises, the patient is standing in front of the mirror and watching his own movement, trying to avoid excessive muscle tension. The rotating movements of the head and neck while performing these exercises can help avoid muscle tension during voice emission.

d Quick alternate inhalations and exhalations during abdominal breathing. The patient blows the exhaled air at a sheet of paper (placed at a $20-$ to $25-\mathrm{cm}$ distance from the lips) to sustain its deflection from the vertical position at the same angle. The patient controls the function of the abdominal muscles by watching his hand placed on the abdomen.

e Relaxed abdominal breathing exercises combined with the voicing of automatic serial speech, e.g. counting, days of the week, sentences and short rhymes. Attention is paid to maintaining correct body posture to reduce unnecessary muscle tension in the throat, larynx and neck areas while voicing.
Additional instructions: before the patient can be admitted to move on to the next stages of the vocal training, he has to acquire an ability of correct breathing support as well as an improvement in the MPT (increase by at least $3 \mathrm{~s}$ ).

$\mathrm{V}$ Relaxation techniques and exercises to reduce muscular tension (1-2 sessions)

a A combination of different relaxation exercises and visualization techniques (e.g. imaginary swallowing of small balls).

b Yawn-sigh approach to reduce excessive vocal muscle tension.

VI Resonance improvement exercises (1-2 sessions)

Principles: all the exercises require appropriate breath support, with the point of vocal tract constriction at the extreme end of the resonators ('making a connection from the abdominal muscles to the lips').

a Murmuring exercises: 'mmm...', 'mo-mo-mo'.

b Sighing exercises: 'hmm-molm-molm-molm...'.

c Voicing at sustained pitch exercises: 'molm-molm...' (slow-fastslow) to feel the vibration of the resonators - experiments with broad and narrow vibrations. Combination of 'molm-molm...' slow-fast-slow and soft-loud-soft with varying rate and intensity, then 'molm-molm .' as spoken phrases with different intonation and varying rate, pitch and loudness.

d Chanting the voiced phrases with plenty of ' $m$ ' consonants on the musical note.

VII Vocal function exercises (3-6 sessions)

Principles: all the vocal exercises should be performed as softly as possible, without a hard glottal attack on initiation of phonation, and with a forward placement of the tone. They require interaction between abdominal contraction and breath support.

a 'Warming-up' exercises (e.g. starting with a sustained vowel /i:/ as long as possible and ending with a maximally sustained /s:/ consonant).

b 'Stretching and contracting' exercises with an open pharynx and lip vibrations, and gliding downwards from the highest to the lowest note and back (Polish standardized phonetic phrases are used).

VIII Carryover exercises (1-2 sessions)

Principles: the exercises involve attempts to transfer the newly acquired behaviours to real-life situations outside the therapy setting, by mimicking different speaking situations, e.g. phone talk. The next step should be to provide a conversation setting outside the therapy room or outside the clinic. The patient gets feedback regarding voice emission from the speech therapist.

The training programme is accomplished when both the patient and the speech therapist regard the effects as satisfying.

IX Control examinations, including videostroboscopy and discussing the effects of the therapy with the patient. The effects of voice therapy were evaluated after a minimum of 2 months since the training had started, after at least 8 logopaedic sessions, and after a significant improvement had been noted by the patient and by the logopaedists. Individual guidelines on the exercises that need to be continued at home under the supervision of a speech therapist.

Principles: the completion of the training programme does not imply a complete recovery of the patient's vocal function. The programme should rather be regarded as a practical demonstration for the patient so that he could still practice correct voice emission and remember the vocal hygiene principles. 


\section{References}

-1 Roy N, Merrill RM, Thibeault S, Parsa R, Gray SD, Smith EM: Prevalence of voice disorders in teachers and general population. J Speech Lang Hear Res 2004;47:281-293.

$\checkmark 2$ Fritzell B: Work-related voice problems: teachers, social workers, lawyers and priests should receive preventive voice training. Lakartidningen 1996;93:1325-1328.

$>3$ Roy N, Merrill RM, Thibeault S, Gray SD, Smith EM: Voice disorders in teachers and the general population: effect on work performance, attendance, and future career choices. J Speech Lang Hear Res 2004;47: 542-551.

4 Smith E, Gray SD, Dove H, Kirchner HL, Heras H: Frequency and effects of teachers' voice problems. J Voice 1997;11:81-87.

5 Urrutikoetxea A, Ispizua A, Matellanes F: Vocal pathology in teachers: a videolaryngostroboscopic study in 1045 teachers. Rev Laryngol Otol Rhinol 1995;116:255-262.

6 Central Statistical Office: Statistical Yearbook of Poland (in Polish). Warsaw, Central Statistical Office, 2005

-7 Śliwińska-Kowalska M, Niebudek-Bogusz E, Fiszer M, Łoś-Spychalska T, Kotyło P, Sznurowska-Przygocka B, Modrzewska $\mathrm{M}$ : The prevalence and risk factors for occupational voice disorders in teachers. Folia Phoniatr Logop 2006;58:85-101.

8 Sliwinska-Kowalska M, Fiszer M, Kotylo P, Ziatkowska E, Stepowska M, Niebudek-Bogusz E: Assessment of voice quality among teachers' college students (in Polish). Med Pr 2000;51:573-580.

-9 MacKenzie K, Millar A, Wilson JA, Sellars C, Deary IJ: Is voice therapy an effective treatment for dysphonia? A randomized controlled trial. BMJ 2001;323:1-6.

-10 Sellars C, Carding PN, Deary IJ, Mackenzie K, Wilson JA: Characterization of effective primary voice therapy for dysphonia. J Laryngol Otol 2002;116:1014-1018.

-11 Kotby MN, El-Sady SR, Basiouny SE: Efficacy of the accent method of voice therapy. J Voice 1991;5:316-320.

-12 Roy N, Gray SD, Simon M, Dove H, CarbinLewis K, Stemple JC: An evaluation of the effects of two treatment approaches for teachers with voice disorders: a prospective randomized clinical trial. J Speech Lang Hear Res 2001;44:286-296.
13 Timmermans B, De Bodt MS, Wuyts FL, Van de Heyning $\mathrm{PH}$ : Training outcome in future professional voice users after 18 months of voice training. Folia Phoniatr Logop 2004; 56:120-129.

14 Śliwińska-Kowalska M, Fiszer M, Kotyło P, Ziatkowska E, Stępowska M, Niebudek-Bogusz E: Effect of voice emission training on the improvement of voice organ function among teachers' college students (in Polish). Med Pr 2002;53:229-232.

15 Koufman JA: Evaluation of laryngeal biomechanics by fiberoptic laryngoscopy; in Rubin JS, Sataloff RT, Korovin GS (eds.): Diagnosis and Treatment of Voice Disorders, ed 2. New York, Thomson Delmar Learning, 2003, pp 171-182.

16 Kooijman PGC, de Jong FICRS, Oudes MJ, Huinck W, van Acht H, Graamans K: Muscular tension and body posture in relation to voice handicap and voice quality in teachers with persistent voice complaints. Folia Phoniatr Logop 2005;57:134-147.

17 Domeracka-Kolodziej A, Maniecka-Aleksandrowicz B, Grzanka A: Voice quality in teachers with occupational voice disorders (in Polish). Otorhinolaryngol Clin Rev 2002; 1:105-112.

18 Rantala L, Vilkman E, Bloigu R: Voice changes during work: subjective complaints and objective measurements for female primary and secondary schoolteachers. J Voice 2002;16:344-355.

19 Jonsdottir V, Laukkanen AM, Vilkman E: Changes in teacher's speech during a working day with and without electric sound amplification. Folia Phoniatr Logop 2002;54: 282-287.

20 Vilkman E: Occupational safety and health aspects of voice and speech professions. Folia Phoniatr Logop 2004;56:220-253.

21 Duffy OM, Hazlett DE: The impact of preventive voice care programs for training teachers: a longitudinal study. J Voice 2004; 18:63-70.

22 Rattenbury HJ, Carding PN, Finn P: Evaluating the effectiveness and efficiency of voice therapy using transnasal flexible laryngoscopy: a randomized controlled trial. J Voice 2004; 18:522-533.
23 Carding PN, Horsley IA, Docherty GJ: The effectiveness of voice therapy for patients with non-organic dyphonia. Clin Otolaryngol 1998;23:310-318.

24 Van Lierde KM, De Ley S, Clement G, De Bodt M, Van Cauwenberge P: Outcome of laryngeal manual therapy in four Dutch adults with persistent moderate-to-severe vocal hyperfunction: a pilot study. J Voice 2004; 18 : 467-474.

25 Altman KW, Atkinson C, Lazarus C: Current and emerging concepts in muscle tension dysphonia: a 30-month review. J Voice 2005;19:261-267.

26 Speyer R, Wieneke G, Kempen PA, Kersing W, Hosseini EG, Dejonckere P: Effects of voice therapy as objectively evaluated by digitized laryngeal stroboscopic imaging. Ann Otol Rhinol Laryngol 2002;111:902-908.

27 Rubin JS, Sataloff RT, Korovin GS: Diagnosis and Treatment of Voice Disorders, ed 2. New York, Thomson Delmar Learning, 2003.

28 Crane SL, Cooper EB: Speech and language: clinician personality variables and clinical effectiveness. J Speech Hear Dis 1983;48: 140-145.

29 Andrews ML, Schmidt CP: Congruence in personality between clinician and client: relationship to ratings of voice treatment. J Voice 1995;3:261-269.

30 Speyer R, Wieneke G, Wijck-Warnaar I, Dejonckere P: Effects of voice therapy on the voice range profiles of dysphonic patients. J Voice 2003; 17:544-556.

31 Pedersen M, Beranova A, Moller S: Dysphonia: medical treatment and a medical voice hygiene advice approach - a prospective randomized pilot study. Eur Arch Otorhinolaryngol 2004;261:312-315.

32 Simberg S, Sala E, Tuomainen J, Sellman J, Rönnemaa AM: The effectiveness of group therapy for students with mild voice disorders: a controlled clinical trial. J Voice 2006; 20:97-109.

-33 Thibeault SL, Merril RM, Roy N, Gray SD, Smith EM: Occupational risk factors associated with voice disorders among teachers. Ann Epidemiol 2004;14:786-792. 